\title{
POPULATION DIVERSITY MAINTENANCE IN BRAIN STORM OPTIMIZATION ALGORITHM
}

\author{
Shi Cheng ${ }^{1}$, Yuhui Shi ${ }^{2}$, Quande Qin ${ }^{3}$, Qingyu Zhang ${ }^{3}$ and Ruibin Bai ${ }^{4}$ \\ ${ }^{1}$ International Doctoral Innovation Centre, The University of Nottingham Ningbo, China \\ Division of Computer Science, University of Nottingham Ningbo, China, \\ Ningbo, 315100, Zhejiang, China \\ ${ }^{2}$ Department of Electrical \& Electronic Engineering, Xi'an Jiaotong-Liverpool University, \\ Suzhou, 215123, Jiangsu, China \\ ${ }^{3}$ Department of Management Science, Shenzhen University, Shenzhen, China, \\ Shenzhen, 518060, Guangdong, China \\ ${ }^{4}$ Division of Computer Science, University of Nottingham Ningbo, China, \\ Ningbo, 315100, Zhejiang, China
}

\begin{abstract}
The convergence and divergence are two common phenomena in swarm intelligence. To obtain good search results, the algorithm should have a balance on convergence and divergence. The premature convergence happens partially due to the solutions getting clustered together, and not diverging again. The brain storm optimization (BSO), which is a young and promising algorithm in swarm intelligence, is based on the collective behavior of human being, that is, the brainstorming process. The convergence strategy is utilized in BSO algorithm to exploit search areas may contain good solutions. The new solutions are generated by divergence strategy to explore new search areas. Premature convergence also happens in the BSO algorithm. The solutions get clustered after a few iterations, which indicate that the population diversity decreases quickly during the search. A definition of population diversity in BSO algorithm is introduced in this paper to measure the change of solutions' distribution. The algorithm's exploration and exploitation ability can be measured based on the change of population diversity. Different kinds of partial reinitialization strategies are utilized to improve the population diversity in BSO algorithm. The experimental results show that the performance of the BSO is improved by part of solutions re-initialization strategies.
\end{abstract}

\section{Introduction}

Optimization, in general, is concerned with finding the "best available" solution(s) for a given problem. Optimization problems can be simply divided into unimodal problems and multimodal problems. As indicated by the name, a unimodal problem has only one optimum solution; on the contrary, a multimodal problem has several or numerous optimum solutions, of which many are local optimal solutions. Galois theory has proved that there is no quintic formula, i.e., the fifth and higher degree equations are not generally solvable by radicals. The iterative method is a powerful tool to solve the fifth and higher degree equations or other difficult functions. Based on the simple rules of iteration, the solution(s) could be improved iteration by iteration, and finally reached to a "good enough" solution. Evolutionary optimization algorithms, or simply the evolutionary algorithms (EAs), are a kind of population-based iterative methods to solve difficult optimization problems. The weakness of 
EAs is generally difficult to find the global optimum solutions for multimodal problems due to the possible occurrence of the premature convergence [1-3]. This balance could be controlled by setting an algorithm's parameters [4].

An optimization problem in $R^{n}$, or simply an optimization problem, is a mapping $f: R^{n} \rightarrow \mathcal{R}^{k}$, where $\mathcal{R}^{n}$ is termed as decision space [5] (or parameter space [6], problem space), and $\mathcal{R}^{k}$ is termed as objective space [7]. Optimization problems can be divided into two categories according to the value of $k$. When $k=1$, this kind of problem is called Single Objective Problems(SOPs), and when $k>1$, this is called Multi-Objective Problems (or Many Objective Optimization, MOO) $[8,9]$.

The evaluation function in optimization, $f(\mathbf{x})$, maps decision variables to objective vectors. Each solution in decision space is associated with a fitness value in objective space. This situation is represented in Fig. 1 for the case $n=3$, and $k=2$.

Evolutionary computation algorithm is inspired from the natural selection process of the physical world, and the swarm intelligence mimics the behaviors of a population of animals/humans in the real world. Both evolutionary computation algorithms and swarm intelligence algorithms can be seen as decentralized systems, and a population of interacting individuals searches in the solution space to optimize a function or goal based on collective adaptation [10].

Swarm intelligence is based on a population of individuals [11]. In swarm intelligence, an algorithm maintains and successively improves a collection of potential solutions until some stopping condition is met. The solutions are initialized randomly in the search space. The search information is propagated through the interaction among solutions. Based on the solutions convergence and divergence, solutions are guided toward the better and better areas.

In swarm intelligence algorithms, there are several solutions which exist at the same time. The premature convergence may happen due to the solution getting clustered together too fast. The population diversity is a measure of exploration and exploitation. Based on the population diversity changing measurement, the state of exploration and exploitation can be obtained. The population diversity def- inition is the first step to give an accurate observation of the search state. Many studies of population diversity in evolutionary computation algorithms and swarm intelligence have been proposed in $[2,12-18]$.

Brain storm optimization (BSO) algorithm is a young and promising swarm intelligence algorithm, which mimics the brainstorming process in which a group of people solves a problem together [19,20]. In a brain storm optimization algorithm, the solutions are divided into several clusters. The solutions being divided into several clusters can be seen as the population diverging into separate species, which are similar to the speciation in the natural selection. The new solutions are generated based on individual(s) in one or two clusters.

BSO algorithm has been utilized to different kinds of problems, such as multimodal optimization [21], multi-objective optimization [22,23]. The parameters in BSO are investigated in [24], the solution clustering is analyzed in [25], the population diversity management is studied in [26]. Many variants of BSO algorithms are proposed. In [27], to reduce the algorithm computational burden, a simple grouping method (SGM) in the grouping operator is introduced to replace the clustering method.

Brain storm optimization algorithm has been utilized into several kinds of real-world problems, such as economic dispatch considering wind power [28], closed-loop BSO algorithm on optimal satellite formation reconfiguration problem [29], predatorCprey BSO algorithm for DC Brushless Motor [30], and quantum-behaved BSO algorithm on solving Loney's Solenoid problem [31].

In this paper, we give a population diversity definition of the brain storm optimization algorithm, and test several partial re-initializing solutions strategies to enhance the population diversity and to help solutions jump out of local optima. The idea behind the re-initialization is to increase the possibility for solutions "jumping out" of local optima, and to keep the ability for the algorithm to find "good enough" solution.

This paper is organized as follows. Section 2 reviews the basic brain storm optimization algorithm. Section 3 gives the definition of population diversity and the diversity maintaining strategies of BSO algorithm. Experiments on unimodal and multi- 


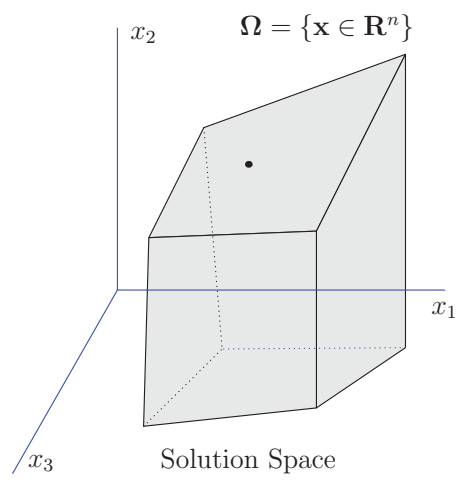

(a)

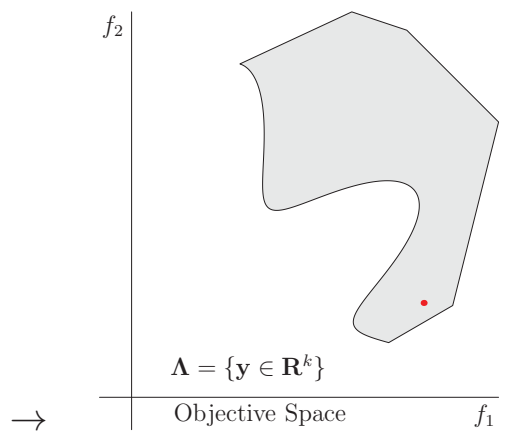

(b)

Figure 1. The mapping from solution space to objective space.

modal benchmark functions are conducted in Section 4. The analysis and discussion of the performance of the BSO algorithm and the population diversity maintaining are given in Section 5. Finally, Section 6 concludes with some remarks and future research directions.

\section{Brain Storm Optimization}

The convergence and divergence are two common phenomena in swarm intelligence. The convergence and divergence information also can be utilized on the search. The framework of divergence and convergence is shown in Fig. 2. The convergence strategy is utilized to explore new possible search region, while the divergence strategy is utilized to exploit existing regions may contains good solutions.

The brain storm optimization algorithm and firework algorithm [32,33] algorithm can be analyzed by the convergence and divergence framework. In BSO algorithm, the random initialized solutions are convergence to different areas. This is a convergence strategy, and the new solutions are generated to diverge the search space. The Firework algorithm [32,33] also utilized convergence and divergence strategies in optimization. Mimicking the explosion of fireworks, the solutions are generated to diverge into large search space. The solutions with good fitness values are selected, which indicated that the solutions are converged to small areas. The convergence and divergence strategies are process iteration by iteration. Based on the iterations of convergence and divergence, the solutions could be clustered to small regions finally.
The BSO algorithm, which is a young and promising algorithm in swarm intelligence, is based on the collective behavior of human being, that is, the brainstorming process $[19,20,34]$. The speciation is a process of natural selection, which means that the population diverging into separate species $[35,36]$. The solutions in BSO are also diverging into several clusters. The new solutions are generated based on the mutation of one individual or interactive of two individuals.

The original BSO algorithm is simple in concept and easy in implementation. The main procedure is given in Algorithm 1. There are three strategies in this algorithm: the solution clustering, new individual generation, and selection [25].

In a brain storm optimization algorithm, the solutions are separated into several clusters. The best solutions of each cluster are kept to the next iteration. New individual can be generated based on one or two individuals in clusters. The exploitation ability is enhanced when the new individual is close to the best solution so far. While the exploration ability is enhanced when the new individual is randomly generated, or generated by individuals in two clusters.

The brain storm optimization algorithm is a kind of search space reduction algorithm [37]; all solutions will get into several clusters eventually. These clusters indicate a problem's local optima. The information of an area contains solutions with good fitness values are propagated from one cluster to another [38]. This algorithm will explore in decision space at first, and the exploration and exploitation will get into a state of equilibrium after iterations. 


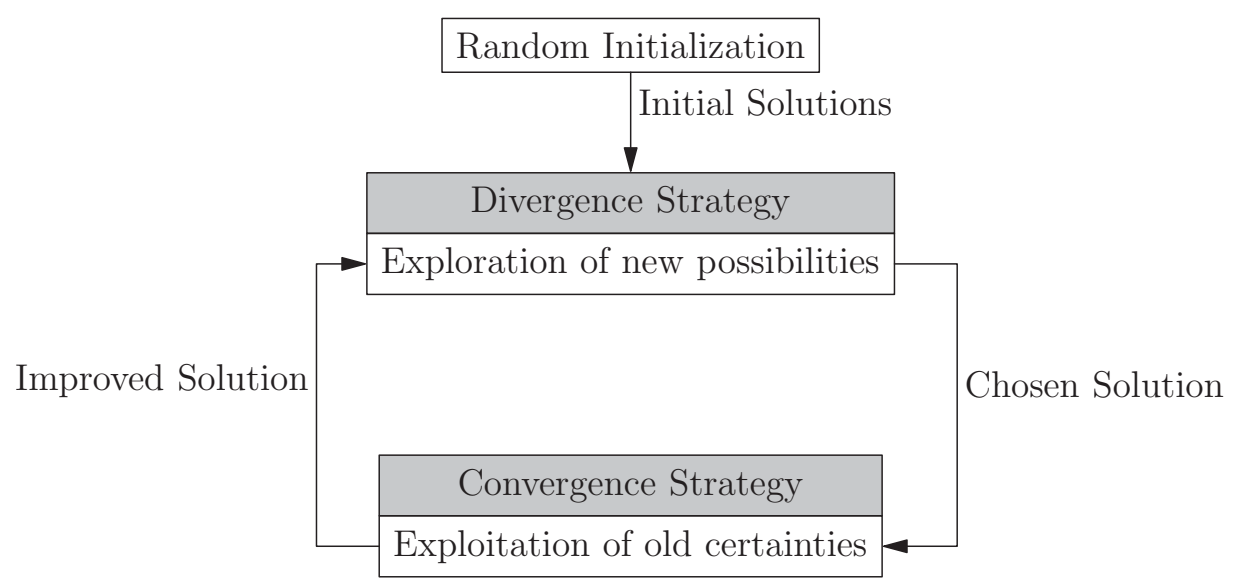

Figure 2. The framework of divergence and convergence in swarm intelligence.

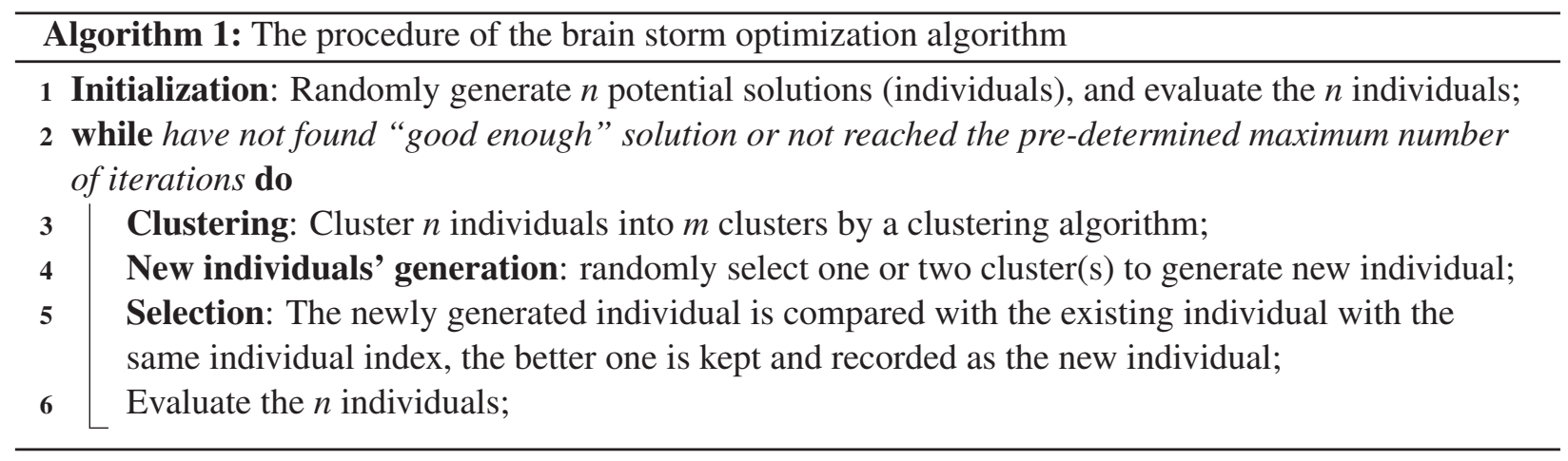

The brain storm optimization algorithm also can be extended to solve multiobjective optimization problems [22,34]. Unlike the traditional multiobjective optimization methods, the brain storm optimization algorithm utilized the objective space information directly. Clusters are generated in the objective space; and for each objective, individuals are clustered in each iteration. The individual, which perform better in most of objectives are kept to the next iteration, and other individuals are randomly selected to keep the diversity of solutions.

\subsection{Solution Clustering}

The aim of solution clustering is to converge the solutions into small regions. Different clustering algorithms can be utilized in the brain storm optimization algorithm. The clustering strategy can be replaced by other convergence method, such as simple grouping method (SGM) [27]. In this paper, the basic $k$-means clustering algorithm is utilized.

Clustering is the process of grouping similar objects together. From the perspective of machine learning, the clustering analysis is sometimes termed as unsupervised learning. There are $N$ points in the given input, $\mathcal{D}=\left\{\mathbf{x}_{i}\right\}_{i=1}^{N}$, the useful and functional patterns can be obtained through the similarity calculation among points [39]. Every solution in the brain storm optimization algorithm is spread in the search space. The distribution of solutions can be utilized to reveal the landscapes of a problem.

The procedure of solution clustering is given in Algorithm 2. The clustering strategy divides individuals into several clusters. This strategy could refine a search area. After many iterations, all solutions may be clustered into a small region. A probability value $p_{\text {clustering }}$ is utilized to control the probability of replacing a cluster center by a randomly generated solution. This could avoid the premature convergence, and help individuals "jump out" of the local optima.

\subsection{New Individual Generation}

The procedure of new individual generation is given in Algorithm 3. A new individual can be gen- 
erated based on one or several individuals or clusters. In the original brain storm optimization algorithm, a probability value $p_{\text {generation }}$ is utilized to determine a new individual being generated by one or two "old" individuals. Generating an individual from one cluster could refine a search region, and it enhances the exploitation ability. On the contrast, an individual, which is generated from two or more clusters, may be far from these clusters. The exploration ability is enhanced in this scenario.

The probability $p_{\text {oneCluster }}$ and probability $p_{\text {twoCluster }}$ are utilized to determine the cluster center or random individual will be chosen in one cluster or two clusters generation case, respectively. In one cluster generation case, the new individual from center or random individual can control the exploitation region. While in several clusters generation case, the random individuals could increase the population diversity of swarm.

The new individuals are generated according to the functions (1) and (2).

$$
\begin{aligned}
& x_{\text {new }}^{i}=x_{\text {old }}^{i}+\xi(t) \times \operatorname{rand}() \\
& \xi(t)=\log \operatorname{sig}\left(\frac{0.5 \times T-t}{c}\right) \times \operatorname{rand}()
\end{aligned}
$$

where $x_{\text {new }}^{i}$ and $x_{\text {old }}^{i}$ are the $i$ th dimension of $x_{\text {new }}$ and $x_{\text {old }}$; and the value $x_{\text {old }}$ is a copy of one individual or the combination of two individuals. The parameter $T$ is the maximum number of iterations, $t$ is the current iteration number, $c$ is a coefficient to change $\log \operatorname{sig}()$ function's slope.

\subsection{Selection}

The selection strategy is utilized to keep good solutions in all individuals. A modified step size and individual generation was proposed in [40]. The step size can be utilized to balance the convergence speed of the algorithm. The better solutions are kept by the selection strategy, while clustering strategy and generation strategy add new solutions into the swarm to keep the diversity for the whole population.

\section{Population Diversity}

The most important factor affecting an optimization algorithm's performance is its ability of "exploration" and "exploitation." Exploration means the ability of a search algorithm to explore different areas of the search space in order to have high probability to find good promising solutions. Exploitation, on the other hand, means the ability to concentrate the search around a promising region in order to refine a candidate solution. A good optimization algorithm should optimally balance the two conflicted objectives $[38,41]$.

In a brain storm optimization algorithm, the solutions are grouped into several clusters. The best solutions of each cluster are kept to the next iteration due to the selection operation. New individual can be generated based on one or two individuals in clusters. The exploitation ability is enhanced when the new individual is close to the best solution so far. While the exploration ability is enhanced when the new individual is randomly generated, or generated by individuals in two clusters.

Population diversity is useful for measuring and dynamically adjusting an algorithm's ability of exploration or exploitation accordingly. In the brain storm optimization algorithm, many solutions are existed at the same time, and these solutions are gathered into several clusters. The solutions may get together into a small region after iterations. The clustering algorithm is difficult to cluster solutions into different group when every solution is within a small region. The algorithm's exploration ability is decreased at this time.

It is important to find a metric to measure the population diversity of solutions in the brain storm optimization algorithm. From the measurement, we can monitor the search of solutions.

\subsection{Population Diversity Definition}

Population diversity is a measurement of solutions' distribution. In [20], proposed $D_{c}, D_{v}$, and $D_{e}$ to measure normalized distance for a cluster, inter-cluster diversity, and information entropy for the population, respectively. Here, in this paper, we define the population diversity given below, which is dimensional-wise and based on the $L_{1}$ norm. 


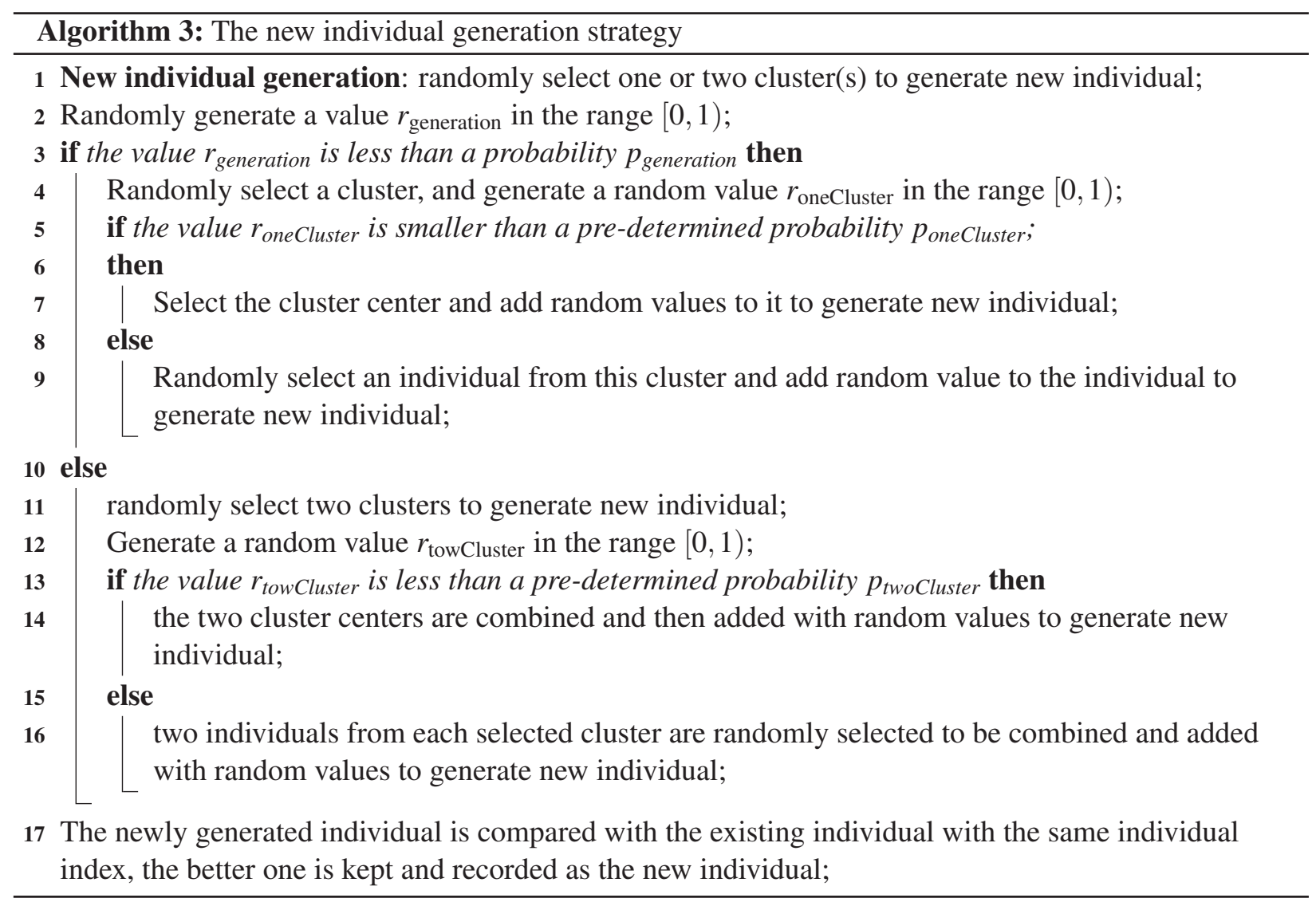

$$
\begin{aligned}
\bar{x}_{j} & =\frac{1}{m} \sum_{i=1}^{m} x_{i j} \\
\operatorname{Div}_{j} & =\frac{1}{m} \sum_{i=1}^{m}\left|x_{i j}-\bar{x}_{j}\right| \\
\operatorname{Div} & =\sum_{j=1}^{n} w_{j} \operatorname{Div}_{j}
\end{aligned}
$$

where $\bar{x}_{j}$ represents the pivot of solutions in dimension $j$, and $\operatorname{Div}_{j}$ measures solution diversity based on $L_{1}$ norm for dimension $j$. Then we define $\overline{\mathbf{x}}=\left[\bar{x}_{1}, \cdots, \bar{x}_{j}, \cdots, \bar{x}_{n}\right], \overline{\mathbf{x}}$ represents the mean of current solutions on each dimension, and Div $=$ $\left[\operatorname{Div}_{1}, \cdots, \operatorname{Div}_{j}, \cdots, \operatorname{Div}_{n}\right]$, which measures solution diversity based on $L_{1}$ norm for each dimension. Div measures the whole group's population diversity.

Without loss of generality, every dimension is considered equally. Setting all weights $w_{j}=\frac{1}{n}$, then the dimension-wise population diversity can be rewritten as:

$$
\operatorname{Div}=\sum_{j=1}^{n} \frac{1}{n} \operatorname{Div}_{j}=\frac{1}{n} \sum_{j=1}^{n} \operatorname{Div}_{j}
$$

\subsection{Population Diversity Maintenance}

Population diversity is a measurement of population state of exploration or exploitation. It illustrates the distribution of solutions. The solutions diverging means that the search is in an exploration state, on the contrary, solutions clustering tightly means that the search is in an exploitation state [42].

The solutions get clustered in search space, and it may not be easy to diverge. The population diversity is decreased when all solutions are clustered into one small region. Many strategies are proposed to enhance the population diversity in evolutionary computation algorithms and swarm intelligence. These strategies include inserting randomly generated individuals, niching $[43,44]$, solutions reinitialization [37,42], or reconstructing the fitness function with the consideration of the age of individuals [45] or the entropy of the population [46].

In this paper, the solutions partial reinitialization is utilized to promote diversity of BSO algorithm. In the brain storm optimization algorithm, the new individual is generated by adding one or two individual(s) with the noise based on equation (1). However, every solution will be very 
similar in each dimension when the solutions get clustered into a small region. The original BSO algorithm may not be easy to escape from local optima. The partial re-initialization in the whole search space could make many solutions diverge into large search areas. The idea behind the reinitialization is to increase possibility for solutions "jumping out" of local optima, and to keep the ability for algorithm to find "good enough" solutions.

Algorithm 4 gives the procedure of the BSO algorithm with re-initialization strategy. After several iterations, part of solutions are re-initialized in whole search space, which increases the possibility of solutions "jumping out" of local optima. According to the number of re-initialized solutions, this strategy can be divided into following categories:

- The number of re-initialized solutions is decreasing during the search process. More than half solutions are re-initialized at the beginning of search, and the number of reinitialized solutions is linearly decreased at each re-initialization. This strategy is to focus on the exploration at first, and the exploitation at the end of the search.

- Part of solutions re-initialized after certain iterations. The number of re-initialized solutions is fixed during the search process. This approach can obtain a great ability of exploration due to the possibility that part of solutions, e.g., half of solutions, will have the chance to escape from local optima.

- The number of re-initialized solutions is increasing during the search process. Less than half solutions are re-initialized at the beginning of search, and the number of reinitialized solutions is linearly increased at each re-initialization. This strategy is to focus on the exploitation at first, and the exploration at the end of the search.

\section{Experimental Study}

Wolpert and Macerady have proved that under certain assumptions no algorithm is better than other one on average for all problems [47]. The aim of the experiment is not to compare the ability or the efficacy of the brain storm optimization algo- rithm with other swarm intelligence algorithms, but the population diversity property of the brain storm optimization algorithm.

\subsection{Benchmark Test Functions and Pa- rameter Setting}

The experiments have been conducted to test the proposed BSO algorithm on the benchmark functions listed in Table 1. Considering the generality, eleven standard benchmark functions were selected, which include five unimodal functions and seven multimodal functions $[48,49]$. All functions are run 50 times to ensure a reasonable statistical result. There are 1500 iterations for 50 dimensional problems in every run. Randomly shifting of the location of optimum is utilized in each dimension for each run.

In all experiments, the brain storm optimization has 200 individuals, and parameters are set as the following, let $p_{\text {clustering }}=0.2, p_{\text {generation }}=0.6$, $p_{\text {oneCluster }}=0.4$ and $p_{\text {twoCluster }}=0.5$. The parameter $k$ in $k$-means algorithm is 20 . The coefficient $c$ is set as 20.0. In the BSO with solution re-initialization, the solutions will be partially re-initialized after each 200 iterations. In the decreasing number of solution re-initialization case, there are 20 solutions are kept at the first time, the number of kept solutions increase 20 at each re-initialization, and 140 solutions are kept at the last time. In the increasing number of solution re-initialization case, there are 180 solutions are kept at the first time, the number of kept solutions increase 20 at each reinitialization, and 60 solutions are kept at the last time.

\subsection{Experimental Results}

Several measures of performance are utilized in this paper. The first is the best fitness value attained after a fixed number of iterations. In our case, we report the best result found after 1500 for 50 dimensional problems. The following measures are the median, the worst and mean value of best fitness values in each run. It is possible that an algorithm will rapidly reach a relatively good result while becoming trapped into a local optimum. These three values give a measure of algorithms' reliability and robustness. 


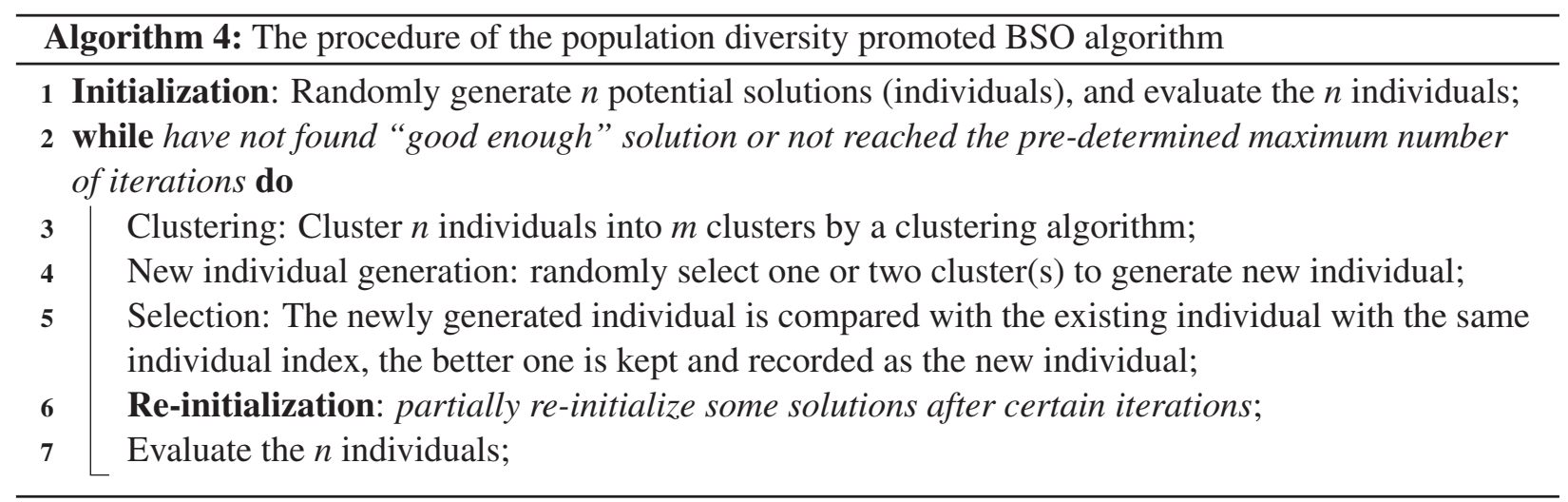

Table 1. The benchmark functions used in experimental study, where $n$ is the dimension of each problem, $\mathbf{z}=(\mathbf{x}-\mathbf{o}), \mathbf{x}=\left[x_{1}, x_{2}, \cdots, x_{n}\right], o_{i}$ is an randomly generated number in problem's search space $S$ and it is different in each dimension, global optimum $\mathbf{x}^{*}=\mathbf{o}, f_{\min }$ is the minimum value of the function, and $S \subseteq \mathcal{R}^{n}$.

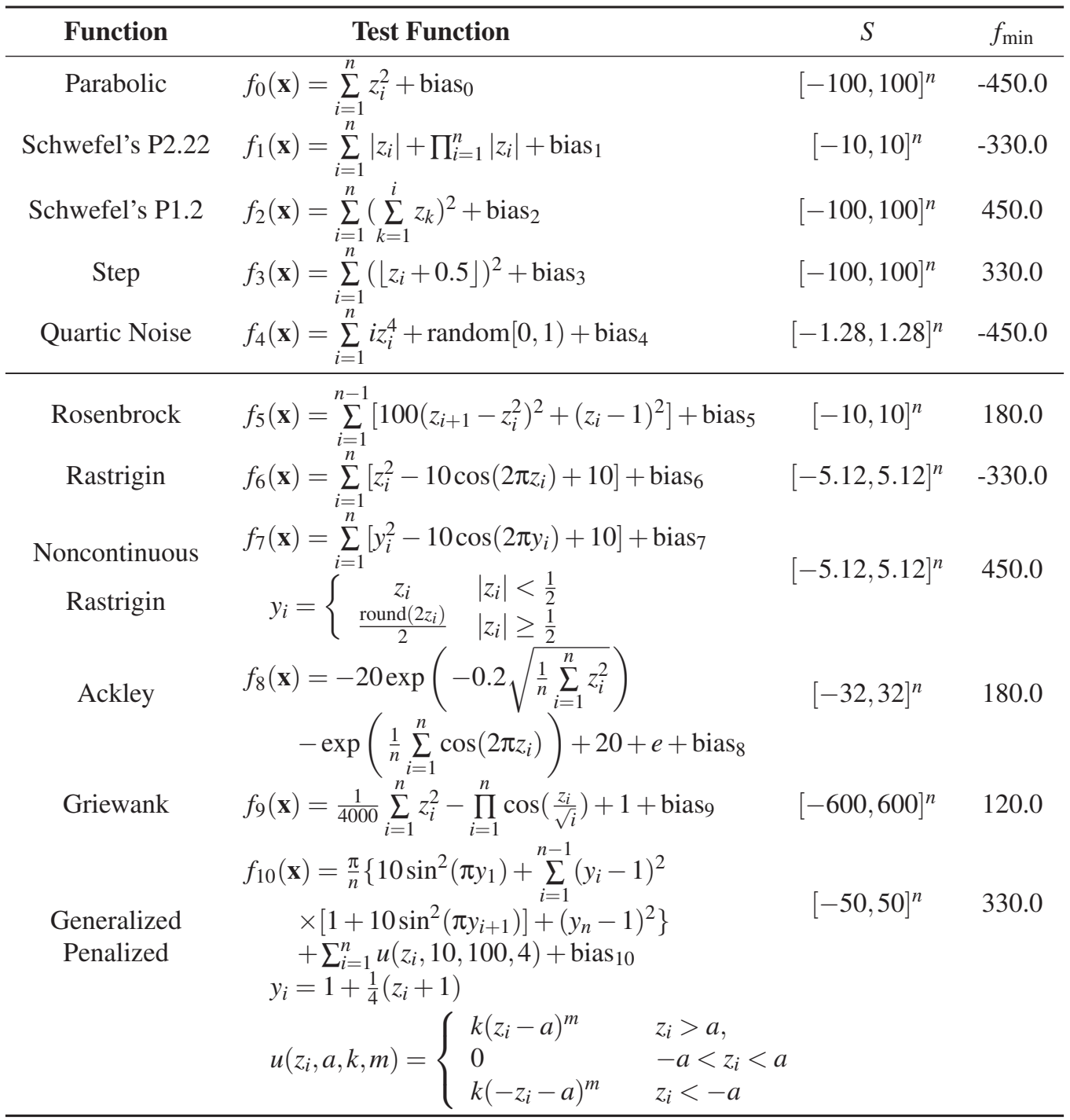


Table 2 gives results of the brain storm optimization algorithm solving unimodal and multimodal problems. The population diversity enhanced BSO performs better than the original BSO for most problems, especially for the unimodal problems.

For traditional algorithms, the multimodal problems are difficult to solve than unimodal problems due to that the multimodal problems have many local optima. However, the brain storm optimization algorithm may be more suitable for multimodal problems. The concept of brain storm optimization algorithm is not to cluster all solutions into one small region, but many regions. From the results, we can find that the original BSO algorithm performs well on the multimodal functions, and the population diversity enhanced BSO algorithm have more improvement in solving unimodal functions than multimodal functions.

\section{Analysis and Discussion}

\subsection{Population Diversity Monitor}

The simulation results give the convergence curves of benchmark functions. Fig. 3 displays the average performance of BSO algorithms solving five unimodal functions. Fig. 4 displays the average performance of BSO algorithms solving six multimodal functions. The brain storm optimization algorithm has a fast convergence at the beginning of search, which indicates that the good search regions can be located after several solution clustering strategies. However, the ability of preventing premature convergence, and "jumping out" of local optima should be improved. Keeping the global search ability, and improving the local search ability should be investigated in the brain storm optimization algorithm.

\subsection{Population Diversity Analysis}

Fig. 5 and Fig. 6 display the population diversity changes during the search process. There are many vibrations of population diversity change in the original BSO solving unimodal functions. The population diversity changes smoothly in the original BSO solving multimodal functions. This may be caused by the different properties of BSO solving unimodal and multimodal functions.
The population diversity is enhanced through the re-initialization strategy. From Fig. 5 and Fig. 6 , we can see that the population diversity change is related to the number of re-initialized solutions. In general, the larger the number of re-initialized solutions is, the smaller the value of population diversity is.

In this experiments, we only tested the reinitialization strategy with fixed number of iterations, and the number of re-initialized solutions is fixed or linear changed. To reveal the relation between the algorithm's performance and the population diversity change, more investigation should be taken on the mechanism of BSO solving different types of problems. The population diversity maintained BSO has promoted the population diversity after certain iterations. The value of population diversity is kept at a large number during the search, this could help the solutions "jump out" a local optima.

\section{Conclusion}

The convergence and divergence are two common phenomena in swarm intelligence. Based on the solutions convergence and divergence, solutions are guided toward the better and better areas. In swarm intelligence algorithms, premature convergence happens partially due to the solutions getting clustered together, and not diverging again. The premature convergence also happens in the brain storm optimization algorithm. To prevent the premature convergence, algorithm's exploration ability and exploitation ability should be balanced during the search.

The population diversity is a measure of exploration and exploitation. Based on the population diversity changing measurement, the state of exploration and exploitation can be obtained. The population diversity definition is the first step to give an accurate observation of the search state. Many approaches have been introduced based on the idea that prevents solutions from clustering too tightly in one region of the search space to achieve great possibility to "jump out" of local optima [50].

In this paper, we introduce a population diversity definition of the brain storm optimization algorithm, and test several kinds of diversity enhanced 
Table 2. Result of brain storm optimization solving unimodal and multimodal benchmark functions. All algorithms are run for 50 times, where "best", "median", "worst", and "mean" indicate the best, median, worst, and mean of the best fitness values for all runs, respectively.

\begin{tabular}{|c|c|c|c|c|c|c|c|}
\hline Func. & $f_{\min }$ & Dim & Best & Median & Worst & Mean & Std. Dev. \\
\hline \multirow{4}{*}{$f_{0}$} & \multirow{4}{*}{-450.0} & original & -283.7674 & 69.3182 & 1011.455 & 128.2867 & 268.266 \\
\hline & & half & -413.8930 & -292.6541 & -73.4257 & -282.6678 & 78.2084 \\
\hline & & decrease & -389.3168 & -296.8813 & 89.42494 & -279.8117 & 93.4272 \\
\hline & & increase & -401.3942 & -222.5723 & 21.8686 & -233.6816 & 88.4352 \\
\hline \multirow{4}{*}{$f_{1}$} & \multirow{4}{*}{-330.0} & original & -329.9999 & -329.9987 & -329.0885 & -329.9615 & 0.16351 \\
\hline & & half & -329.9999 & -329.9975 & -329.9844 & -329.9968 & 0.00311 \\
\hline & & decrease & -329.9999 & -329.9978 & -329.9872 & -329.9969 & 0.00297 \\
\hline & & increase & -329.9999 & -329.9982 & -329.9907 & -329.9974 & 0.00227 \\
\hline \multirow{4}{*}{$f_{2}$} & \multirow{4}{*}{450.0} & original & 1674.185 & 3469.0662 & 6521.9105 & 3715.998 & 1155.126 \\
\hline & & half & 1236.369 & 1715.393 & 2518.608 & 1770.651 & 365.281 \\
\hline & & decrease & 1013.162 & 1734.731 & 2432.571 & 1682.709 & 311.921 \\
\hline & & increase & 1302.540 & 2088.135 & 2941.036 & 2078.788 & 417.398 \\
\hline \multirow{4}{*}{$f_{3}$} & \multirow{4}{*}{330.0} & original & 1461 & 1989 & 4036 & 2121.96 & 450.6883 \\
\hline & & half & 765 & 1122 & 1548 & 1110.22 & 163.962 \\
\hline & & decrease & 785 & 1095 & 1536 & 1086.92 & 173.0487 \\
\hline & & increase & 875 & 1185 & 1768 & 1221.74 & 205.0707 \\
\hline \multirow{4}{*}{$f_{4}$} & \multirow{4}{*}{-450.0} & original & -449.9989 & -449.9966 & -449.9933 & -449.9963 & 0.00116 \\
\hline & & half & -449.9983 & -449.9960 & -449.9934 & -449.9960 & 0.00114 \\
\hline & & decrease & -449.9978 & -449.9955 & -449.9922 & -449.9955 & 0.00130 \\
\hline & & increase & -449.9982 & -449.9961 & -449.9928 & -449.9960 & 0.00132 \\
\hline \multirow{3}{*}{$f_{5}$} & \multirow{4}{*}{180.0} & original & 221.5290 & 227.5745 & 288.2411 & 232.1447 & 15.4617 \\
\hline & & half & 219.0803 & 227.5494 & 389.8904 & 239.6247 & 33.4026 \\
\hline & & decrease & 221.2358 & 227.3915 & 360.8707 & 237.8019 & 26.1117 \\
\hline \multirow{5}{*}{$f_{6}$} & & increase & 218.9889 & 227.7686 & 336.3135 & 240.7103 & 25.7083 \\
\hline & \multirow{4}{*}{-330.0} & original & -300.1512 & -265.3277 & -224.5344 & -264.9098 & 17.7013 \\
\hline & & half & -301.1461 & -271.2974 & -198.6657 & -267.5166 & 20.5183 \\
\hline & & decrease & -295.1764 & -263.3378 & -190.7060 & -260.3728 & 22.7473 \\
\hline & & increase & -294.1814 & -271.2974 & -225.5294 & -270.0637 & 16.5337 \\
\hline \multirow{3}{*}{$f_{7}$} & \multirow{3}{*}{450.0} & original & 482 & 526 & 619 & 528.68 & 27.3447 \\
\hline & & half & 487 & 532 & 592 & 528.04 & 20.8891 \\
\hline & & decrease & 487 & 528 & 606 & 530.58 & 25.4684 \\
\hline \multirow{5}{*}{$f_{8}$} & \multirow{5}{*}{180.0} & increase & 486 & 528 & 581 & 526.28 & 20.9074 \\
\hline & & original & 188.2361 & 190.7374 & 192.2957 & 190.6498 & 0.84468 \\
\hline & & half & 186.9072 & 190.3279 & 192.1137 & 190.1500 & 1.13627 \\
\hline & & decrease & 187.4559 & 190.5704 & 191.8832 & 190.2716 & 1.07905 \\
\hline & & increase & 186.9843 & 190.6153 & 192.3914 & 190.4371 & 1.16226 \\
\hline \multirow{3}{*}{$f_{9}$} & \multirow{4}{*}{120.0} & original & 129.8876 & 134.4022 & 142.2110 & 134.6174 & 2.88916 \\
\hline & & half & 124.2548 & 126.2922 & 130.5381 & 126.3547 & 1.28784 \\
\hline & & decrease & 124.3876 & 126.1242 & 129.6540 & 126.1661 & 1.14855 \\
\hline \multirow{5}{*}{$f_{10}$} & & increase & 123.8568 & 127.1556 & 131.5378 & 127.4312 & 1.65378 \\
\hline & \multirow{4}{*}{330.0} & original & 332.1512 & 336.5663 & 344.5289 & 337.1611 & 2.89567 \\
\hline & & half & 332.5938 & 336.9778 & 344.0822 & 337.3973 & 2.97637 \\
\hline & & decrease & 332.1948 & 336.2336 & 345.7965 & 337.0947 & 2.84417 \\
\hline & & increase & 332.2791 & 336.70007 & 343.7704 & 337.2055 & 2.67052 \\
\hline
\end{tabular}




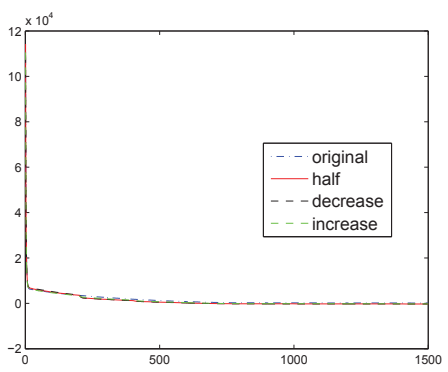

(a) Parabolic $f_{0}$

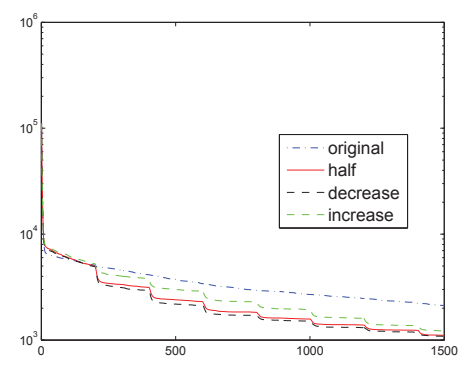

(d) Step $f_{3}$

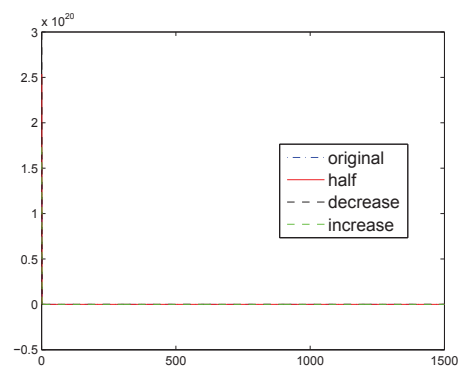

(b) Schwefel's P2.22 $f_{1}$

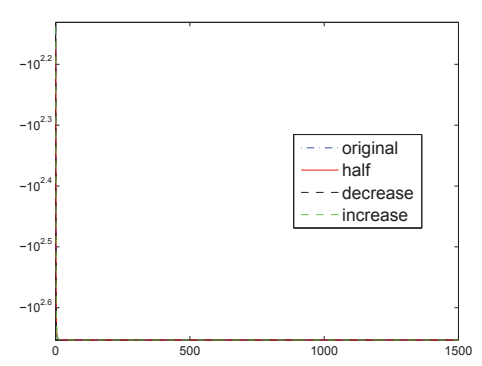

(e) Quartic Noise $f_{4}$

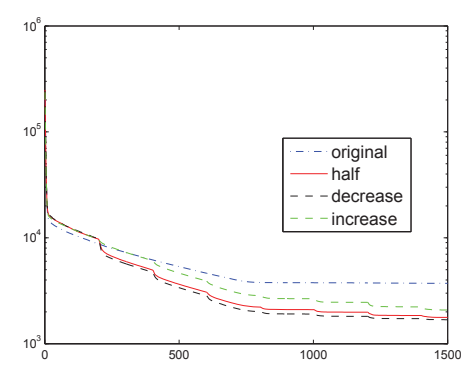

(c) Schwefel's P1.2 $f_{2}$

Figure 3. The average performance of the brain storm optimization algorithm solving unimodal functions.

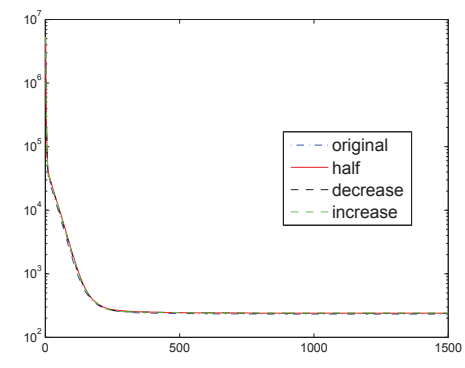

(a) Rosenbrock $f_{5}$

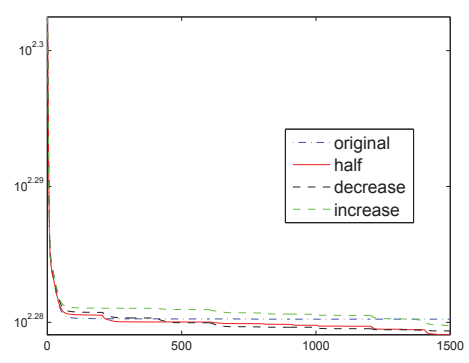

(d) Ackley $f_{8}$

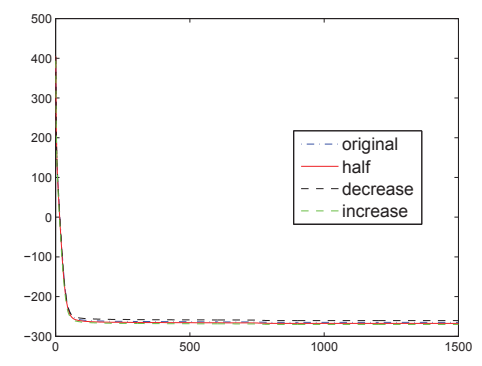

(b) Rastrigin $f_{6}$

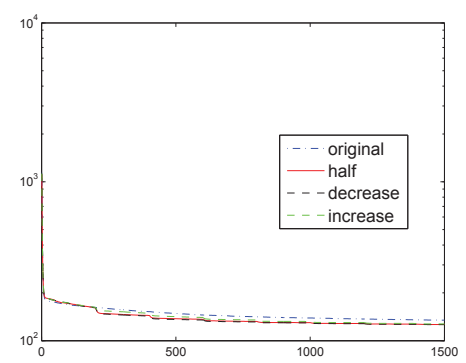

(e) Griewank $f_{9}$

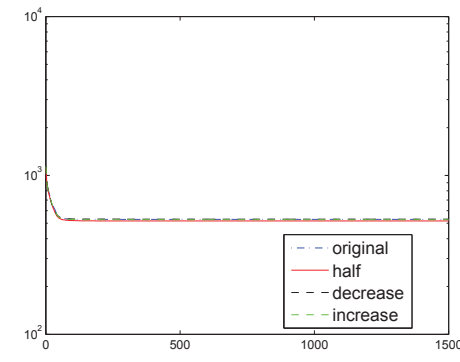

(c) Noncontinuous Rastrigin $f_{7}$

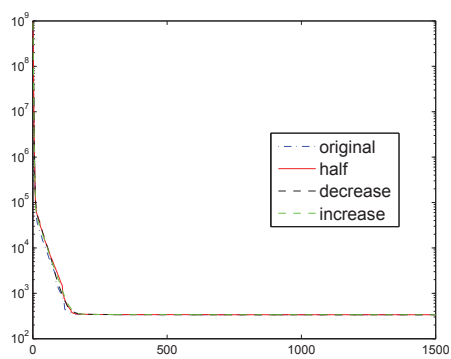

(f) Generalized Penalized $f_{10}$

Figure 4. The average performance of the brain storm optimization algorithm solving multimodal functions. 


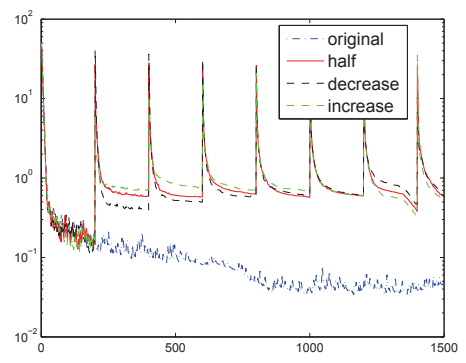

(a) Parabolic $f_{0}$

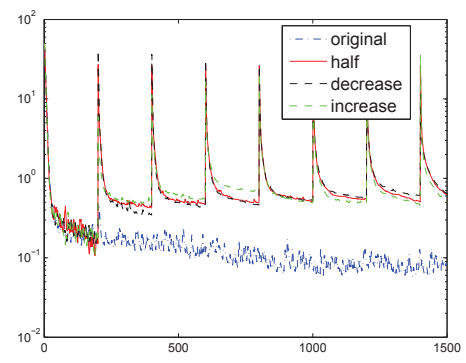

(d) Step $f_{3}$

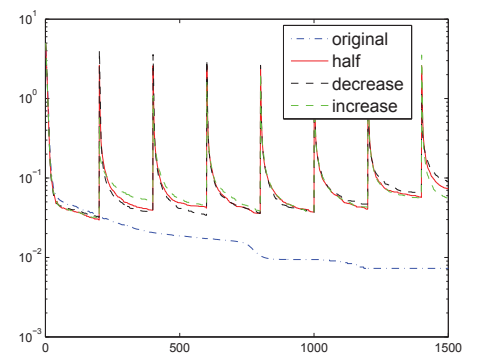

(b) Schwefel's P2.22 $f_{1}$

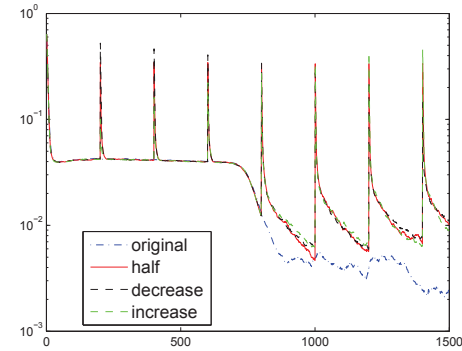

(e) Quartic Noise $f_{4}$

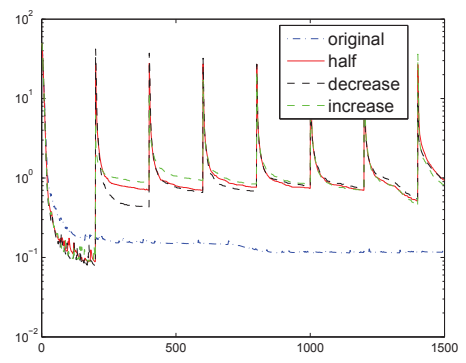

(c) Schwefel's P1.2 $f_{2}$

Figure 5. The population diversity monitor of the brain storm optimization algorithm solving unimodal functions.

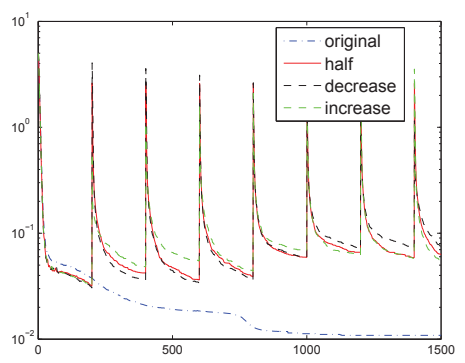

(a) Rosenbrock $f_{5}$

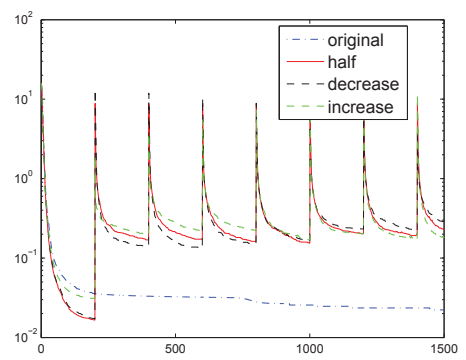

(d) Ackley $f_{8}$

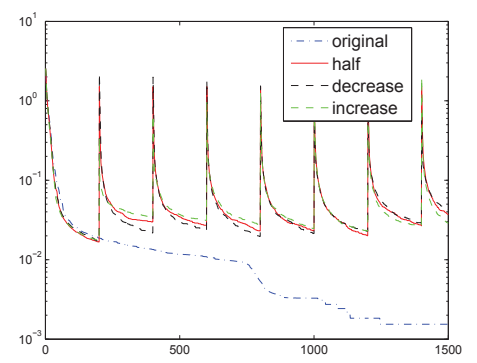

(b) Rastrigin $f_{6}$

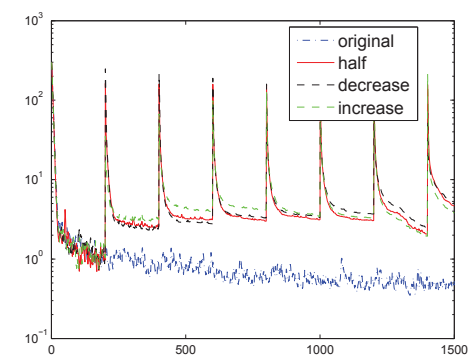

(e) Griewank $f_{9}$

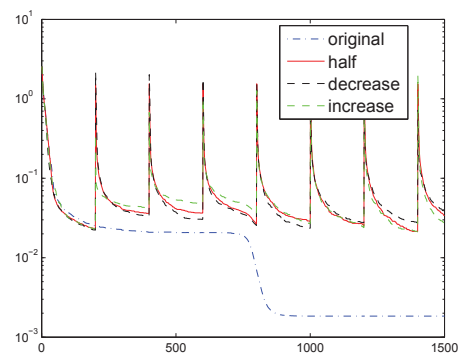

(c) Noncontinuous Rastrigin $f_{7}$

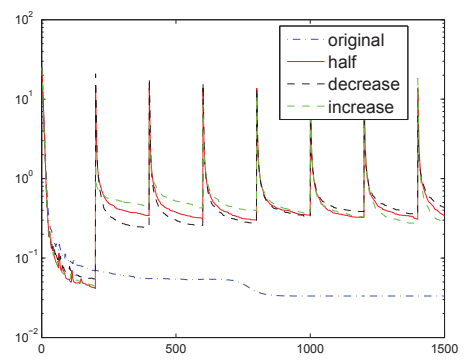

(f) Generalized Penalized $f_{10}$

Figure 6. The population diversity monitor of the brain storm optimization algorithm solving multimodal functions. 
strategies to help solutions jump out of local optima. The experimental study shows that the performance of optimization is improved by the population diversity enhancement. The population diversity also should be monitored in the brain storm optimization algorithm solving multiobjective problems. The relationship between the population diversity changes and the performance of BSO algorithm, and the properties of population diversity changes with different problems also needs more analysis. In general, the brain storm optimization algorithm is a young and promising algorithm; there are many fields which are under investigation.

\section{Acknowledgment}

This work was carried out at the International Doctoral Innovation Centre (IDIC). The authors acknowledge the financial support from Ningbo Education Bureau, Ningbo Science and Technology Bureau, China's MOST and The University of Nottingham. This work is also partially supported by National Natural Science Foundation of China under grant No.71240015, 71402103, 61273367; and Ningbo Science \& Technology Bureau (Science and Technology Project No.2012B10055). This is an extension from CEC 2014 conference paper "Maintaining Population Diversity in Brain Storm Optimization Algorithm" [26].

\section{References}

[1] K. A. De Jong, "An analysis of the behavior of a class of genetic adaptive systems," Ph.D. dissertation, Department of Computer and Communication Sciences, University of Michigan, August 1975.

[2] M. L. Mauldin, "Maintaining diversity in genetic search," in Proceedings of the National Conference on Artificial Intelligence (AAAI 1984), August 1984, pp. 247-250.

[3] D. E. Goldberg, Genetic Algorithms in Search, Optimization and Machine Learning. Boston, MA, USA: Addison-Wesley Longman Publishing Co., Inc., 1989.

[4] Á. E. Eiben, R. Hinterding, and Z. Michalewicz, "Parameter control in evolutionary algorithms," IEEE Transactions on Evolutionary Computation, vol. 3, no. 2, pp. 124-141, July 1999.

[5] S. F. Adra, T. J. Dodd, I. A. Griffin, and P. J. Fleming, "Convergence acceleration operator for mul- tiobjective optimization," IEEE Transactions on Evolutionary Computation, vol. 12, no. 4, pp. 825847, August 2009.

[6] Y. Jin and B. Sendhoff, "A systems approach to evolutionary multiobjective structural optimization and beyond," IEEE Computational Intelligence Magazine, vol. 4, no. 3, pp. 62-76, August 2009.

[7] R. K. Sundaram, A First Course in Optimization Theory. Cambridge University Press, 1996.

[8] R. C. Purshouse and P. J. Fleming, "On the evolutionary optimization of many conflicting objectives," IEEE Transactions on Evolutionary Computation, vol. 11 , no. 6, pp. 770-784, December 2007.

[9] S. F. Adra and P. J. Fleming, "Diversity management in evolutionary many-objective optimization," IEEE Transactions on Evolutionary Computation, vol. 15, no. 2, pp. 183-195, April 2011.

[10] A. Engelbrecht, X. Li, M. Middendorf, and L. M. Gambardella, "Editorial special issue: Swarm intelligence," IEEE Transactions on Evolutionary Computation, vol. 13, no. 4, pp. 677-680, August 2009.

[11] J. Kennedy, R. Eberhart, and Y. Shi, Swarm Intelligence. Morgan Kaufmann Publisher, 2001.

[12] E. K. Burke, S. Gustafson, and G. Kendall, "A survey and analysis of diversity measures in genetic programming," in Proceedings of the Genetic and Evolutionary Computation Conference (GECCO 2002). San Francisco, CA, USA: Morgan Kaufmann Publishers Inc., 2002, pp. 716-723.

[13] Y. Shi and R. Eberhart, "Population diversity of particle swarms," in Proceedings of the 2008 Congress on Evolutionary Computation (CEC2008), 2008, pp. 1063-1067.

[14] _ , "Monitoring of particle swarm optimization," Frontiers of Computer Science, vol. 3, no. 1, pp. 31-37, March 2009.

[15] S. Cheng and Y. Shi, "Diversity control in particle swarm optimization," in Proceedings of 2011 IEEE Symposium on Swarm Intelligence (SIS 2011), Paris, France, April 2011, pp. 110-118.

[16] S. Cheng, Y. Shi, and Q. Qin, "Experimental Study on Boundary Constraints Handling in Particle Swarm Optimization: From Population Diversity Perspective," International Journal of Swarm Intelligence Research (IJSIR), vol. 2, no. 3, pp. 4369, July-September 2011.

[17] S. Cheng, "Population diversity in particle swarm optimization: Definition, observation, control, and application," Ph.D. dissertation, Department of 
Electrical Engineering and Electronics, University of Liverpool, May 2013.

[18] S. Cheng, Y. Shi, and Q. Qin, "A study of normalized population diversity in particle swarm optimization," International Journal of Swarm Intelligence Research (IJSIR), vol. 4, no. 1, pp. 1-34, January-March 2013.

[19] Y. Shi, "Brain storm optimization algorithm," in Advances in Swarm Intelligence, ser. Lecture Notes in Computer Science, Y. Tan, Y. Shi, Y. Chai, and G. Wang, Eds. Springer Berlin/Heidelberg, 2011, vol. 6728, pp. 303-309.

[20] — - "An optimization algorithm based on brainstorming process," International Journal of Swarm Intelligence Research (IJSIR), vol. 2, no. 4, pp. 3562, October-December 2011.

[21] X. Guo, Y. Wu, and L. Xie, "Modified brain storm optimization algorithm for multimodal optimization," in Advances in Swarm Intelligence, ser. Lecture Notes in Computer Science, Y. Tan, Y. Shi, and C. A. C. Coello, Eds. Springer International Publishing, 2014, vol. 8795, pp. 340-351.

[22] J. Xue, Y. Wu, Y. Shi, and S. Cheng, "Brain storm optimization algorithm for multi-objective optimization problems," in Advances in Swarm Intelligence, ser. Lecture Notes in Computer Science, Y. Tan, Y. Shi, and Z. Ji, Eds. Springer Berlin / Heidelberg, 2012, vol. 7331, pp. 513-519.

[23] L. Xie and Y. Wu, "A modified multi-objective optimization based on brain storm optimization algorithm," in Advances in Swarm Intelligence, ser. Lecture Notes in Computer Science, Y. Tan, Y. Shi, and C. Coello, Eds. Springer International Publishing, 2014, vol. 8795, pp. 328-339.

[24] Z.-H. Zhan, W.-N. Chen, Y. Lin, Y.-J. Gong, Y. long Li, and J. Zhang, "Parameter investigation in brain storm optimization," in 2013 IEEE Symposium on Swarm Intelligence (SIS), April 2013, pp. 103-110.

[25] S. Cheng, Y. Shi, Q. Qin, and S. Gao, "Solution clustering analysis in brain storm optimization algorithm," in Proceedings of The 2013 IEEE Symposium on Swarm Intelligence, (SIS 2013). Singapore: IEEE, 2013, pp. 111-118.

[26] S. Cheng, Y. Shi, Q. Qin, T. O. Ting, and R. Bai, "Maintaining population diversity in brain storm optimization algorithm," in Proceedings of 2014 IEEE Congress on Evolutionary Computation, (CEC 2014). Beijing, China: IEEE, 2014, pp. 3230-3237.
[27] Z. hui Zhan, J. Zhang, Y. hui Shi, and H. lin Liu, "A modified brain storm optimization," in Evolutionary Computation (CEC), 2012 IEEE Congress on, June 2012, pp. 1-8.

[28] H. Jadhav, U. Sharma, J. Patel, and R. Roy, "Brain storm optimization algorithm based economic dispatch considering wind power," in 2012 IEEE International Conference on Power and Energy (PECon 2012), Kota Kinabalu, Malaysia, December 2012, pp. 588-593.

[29] C. Sun, H. Duan, and Y. Shi, "Optimal satellite formation reconfiguration based on closed-loop brain storm optimization," IEEE Computational Intelligence Magazine, vol. 8, no. 4, pp. 39-51, November 2013.

[30] H. Duan, S. Li, and Y. Shi, "Predatorcprey brain storm optimization for dc brushless motor," IEEE Transactions on Magnetics, vol. 49, no. 10, pp. 5336-5340, October 2013.

[31] H. Duan and C. Li, "Quantum-behaved brain storm optimization approch to solving loney's solenoid problem," IEEE Transactions on Magnetics, p. in press, 2014.

[32] Y. Tan and Y. Zhu, "Fireworks algorithm for optimization," in Advances in Swarm Intelligence, ser. Lecture Notes in Computer Science, Y. Tan, Y. Shi, and K. C. Tan, Eds. Springer Berlin Heidelberg, 2010, vol. 6145, pp. 355-364.

[33] S. Zheng, A. Janecek, and Y. Tan, "Enhanced fireworks algorithm," in 2013 IEEE Congress on Evolutionary Computation (CEC), June 2013, pp. 2069-2077.

[34] Y. Shi, J. Xue, and Y. Wu, "Multi-objective optimization based on brain storm optimization algorithm," International Journal of Swarm Intelligence Research (IJSIR), vol. 4, no. 3, pp. 1-21, July-September 2013.

[35] C. Darwin, On the Origin of Species by Means of Natural Selection, or the Preservation of Favoured Races in the Struggle for Life, 5th ed. London: John Murray, 1869.

[36] M. Affenzeller, S. Winkler, S. Wagner, and A. Beham, Genetic Algorithms and Genetic Programming: Modern Concepts and Practical Applications, ser. Numerical Insights, A. Sydow, Ed. Chapman \& Hall/CRC Press, 2009, vol. 6.

[37] S. Cheng, Y. Shi, and Q. Qin, "Dynamical exploitation space reduction in particle swarm optimization for solving large scale problems," in Proceedings of 2012 IEEE Congress on Evolutionary Computation, (CEC 2012). Brisbane, Australia: IEEE, 2012, pp. 3030-3037. 
[38] _ _ "Population diversity based study on search information propagation in particle swarm optimization," in Proceedings of 2012 IEEE Congress on Evolutionary Computation, (CEC 2012). Brisbane, Australia: IEEE, 2012, pp. 1272-1279.

[39] K. P. Murphy, Machine Learning: A Probabilistic Perspective, ser. Adaptive computation and machine learning series. Cambridge, Massachusetts: The MIT Press, 2012.

[40] D. Zhou, Y. Shi, and S. Cheng, "Brain storm optimization algorithm with modified step-size and individual generation," in Advances in Swarm Intelligence, ser. Lecture Notes in Computer Science, Y. Tan, Y. Shi, and Z. Ji, Eds. Springer Berlin / Heidelberg, 2012, vol. 7331, pp. 243-252.

[41] S. Cheng, Y. Shi, and Q. Qin, "Population diversity of particle swarm optimizer solving single and multi-objective problems," International Journal of Swarm Intelligence Research (IJSIR), vol. 3, no. 4, pp. 23-60, 2012.

[42] — , "Promoting diversity in particle swarm optimization to solve multimodal problems," in $\mathrm{Neu}$ ral Information Processing, ser. Lecture Notes in Computer Science, B.-L. Lu, L. Zhang, and J. Kwok, Eds. Springer Berlin / Heidelberg, 2011, vol. 7063, pp. 228-237.

[43] W. Cedeño and V. R. Vemuri, "On the use of niching for dynamic landscapes," in Proceedings of 1997 IEEE Congress on Evolutionary Computation, (CEC 1997). IEEE, 1997, pp. 361-366.

[44] A. Della Cioppa, C. De Stefano, and A. Marcelli, "Where are the niches? dynamic fitness sharing," IEEE Transactions on Evolutionary Computation, vol. 11, no. 4, pp. 453-465, August 2007.
[45] A. Ghosh, S. Tsutsui, and H. Tanaka, "Function optimization in nonstationary environment using steady state genetic algorithms with aging of individuals," in Proceedings of 1998 IEEE Congress on Evolutionary Computation, (CEC 1998). IEEE, 1998, pp. 666-671.

[46] Y. Jin and B. Sendhoff, "Constructing dynamic optimization test problems using the multi-objective optimization concept," in Applications of Evolutionary Computing, ser. Lecture Notes in Computer Science, G. R. Raidl, S. Cagnoni, J. Branke, D. W. Corne, R. Drechsler, Y. Jin, C. G. Johnson, P. Machado, E. Marchiori, F. Rothlauf, G. D. Smith, and G. Squillero, Eds. Springer Berlin / Heidelberg, 2004, vol. 3005, pp. 525-536.

[47] D. H. Wolpert and W. G. Macready, "No free lunch theorems for optimization," IEEE Transactions on Evolutionary Computation, vol. 1, no. 1, pp. 6782, April 1997.

[48] X. Yao, Y. Liu, and G. Lin, "Evolutionary programming made faster," IEEE Transactions on Evolutionary Computation, vol. 3, no. 2, pp. 82-102, July 1999.

[49] J. J. Liang, A. K. Qin, P. N. Suganthan, and S. Baskar, "Comprehensive learning particle swarm optimizer for global optimization of multimodal functions," IEEE Transactions on Evolutionary Computation, vol. 10, no. 3, pp. 281-295, June 2006.

[50] T. Blackwell and P. Bentley, "Don't push me! collision-avoiding swarms," in Proceedings of The Fourth Congress on Evolutionary Computation (CEC 2002), May 2002, pp. 1691-1696. 